\title{
Editorial
}

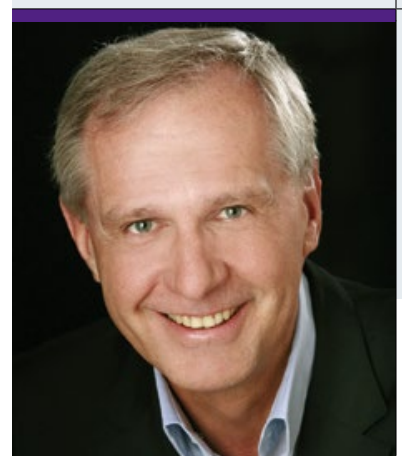

„Die vielbeschworene Wiedergeburt des Hormonersatzes

wird mit einer bemerkenswerten Zurückhaltung propagiert."

Prof. Dr. med. Bernd Kleine-Gunk

Leiter der Gynäkologie am Metropol Medical Center, Nürnberg, und Präsident der Deutschen Gesellschaft für Prävention und Anti-Aging-Medizin (GSAAM)

\section{Die Renaissance der HRT - auch als Anti-Aging Therapie}

A uch nach 15 Jahren sitzt der WHI-Schock noch immer tief. Die Fehler der Studie sind zwar seit Langem umfassend analysiert, die "Renaissance der HRT" wird auf sämtlichen Kongressen verkündet, und die Autoren der WHI-Studie selbst haben sich inzwischen für die Fehlinterpretationen entschuldigt. Dennoch wird die vielbeschworene Wiedergeburt des Hormonersatzes mit einer bemerkenswerten Zurückhaltung propagiert. "So wenig wie nötig und das so kurz wie möglich“" lautet noch immer die gängige Formel, wenn es um die Gabe von Hormonen nach den Wechseljahren geht. Der erste Halbsatz ist zweifellos richtig. Der zweite nicht.

Sowohl neuere Studien wie die Early Versus Late Postmenopausal Treatment With Estradiol-Study (ELITE) und die Kronos Early Estrogen Prevention Study (KEEPS) als auch die Reevaluation der WHIStudie zeigen: Die Hormonersatztherapie ist nicht nur eine wirksame Behandlung gegen psychovegetative klimakterische Beschwerden, sie ist auch eine effektive präventive Maßnahme gegen wichtige Organerkrankungen. Das kardiovaskuläre Risiko sinkt, der Osteoporose wird vorgebeugt und selbst die Alzheimer-Demenz wird verzögert (siehe hierzu auch den CME-Beitrag auf Seite 32 ff.). Immer vorausgesetzt, das sogenannte zeitliche Fenster (,window of opportunity“) wird eingehalten. In der Zeit zwischen fünfzig und sechzig Jahren überwiegt der gesundheitliche Nutzen einer Hormonersatztherapie meist ganz klar die geringen Risiken.

Nun sind zehn Jahre ja alles andere als eine kurze Zeit. Statt gebetsmühlenartig das Mantra „So kurz wie möglich" "zu wiederholen, sollten wir also im Gegenteil alles daransetzen, unsere Patientinnen zu motivieren, die Spanne von zehn Jahren auch wirklich auszunutzen. Es gibt in der Medizin nicht viele Beispiele dafür, dass durch eine einzelne pharmakologische Intervention gleich drei wichtigen altersabhängigen Erkrankungen (Arteriosklerose, Osteoporose und Demenz) vorgebeugt wird.

Ich nenne so etwas eine Anti-Aging-Therapie, auch wenn ich weiß, dass sich viele mit dem Begriff immer noch schwer tun. Man kann dafür sicherlich auch andere Bezeichnungen finden. Wie wäre es zum Beispiel mit „Präventive Endokrinologie“?

Letztlich kommt es aber nicht auf Begrifflichkeiten an, sondern auf die praktische Umsetzung. Das bedeutet: Ein klares Ja zur Hormonersatztherapie. Und das mindestens für zehn Jahre.

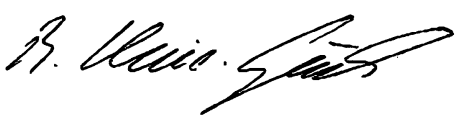

